Article

\title{
Evaluation of GVPC and BCYE Media for Legionella Detection and Enumeration in Water Samples by ISO 11731: Does Plating on BCYE Medium Really Improve Yield?
}

\author{
Maria Scaturro 1(D, Elisa Poznanski ${ }^{2}$, Mariarosaria Mupo ${ }^{2}$, Paola Blasior ${ }^{2}$, Margit Seeber ${ }^{2}$, \\ Anna-Maria Prast ${ }^{2}$, Elisa Romanin ${ }^{2}$, Antonietta Girolamo ${ }^{1}$, Maria Cristina Rota ${ }^{1}$ (D), \\ Antonino Bella ${ }^{1}$ (D), Maria Luisa Ricci ${ }^{1, *,+}$ and Alberta Stenico ${ }^{2, *, \dagger}$ \\ 1 Department of Infectious Diseases, Istituto Superiore di Sanità, Viale Regina Elena 299, 00161 Rome, Italy; \\ maria.scaturro@iss.it (M.S.); antonietta.girolamo@iss.it (A.G.); mariacristina.rota@iss.it (M.C.R.); \\ antonino.bella@iss.it (A.B.) \\ 2 Biological Laboratory, Provincial Agency for the Environment and Climate Protection, \\ 39055 Laives (Bolzano), Italy; elisa.poznanski@provincia.bz.it (E.P.); \\ mariarosaria.mupo@provincia.bz.it (M.M.); paola.blasior@provincia.bz.it (P.B.); \\ margit.seeber@provincia.bz.it (M.S.); anna-maria.prast@provincia.bz.it (A.-M.P.); \\ elisa.romanin@provincia.bz.it (E.R.) \\ * Correspondence: marialuisa.ricci@iss.it (M.L.R.); alberta.stenico@provincia.bz.it (A.S.); \\ Tel.: +39-06-4990-2856 (M.L.R.); +39-04-7195-0431 (A.S.) \\ + These authors contributed equally to this work.
}

Received: 28 August 2020; Accepted: 15 September 2020; Published: 16 September 2020

\begin{abstract}
Legionella spp are the causative agents of Legionnaires' diseases, which is a pneumonia of important public health concern. Ubiquitous freshwater and soil inhabitants can reach man-made water systems and cause illness. Legionella enumeration and quantification in water systems is crucial for risk assessment and culture examination is the gold standard method. In this study, Legionella recovery from potable water samples, at presumably a low concentration of interfering microorganisms, was compared by plating on buffered charcoal yeast extract (BCYE) and glycine, vancomycin, polymyxin B, cycloheximide (GVPC) Legionella agar media, according to the International Standard Organization (ISO) 11731: 2017. Overall, 556 potable water samples were analyzed and 151 (27.1\%) were positive for Legionella. Legionella grew on both BCYE and GVPC agar plates in 85/151 (56.3\%) water samples, in 65/151 (43\%) on only GVPC agar plates, and in 1/151 (0.7\%) on only BCYE agar plates. In addition, GVPC medium identified Legionella species other than pneumophila in six more samples as compared with the culture on BCYE. Although the medians of colony forming units per liter (CFU/L) detected on the BCYE and GVPC agar plates were 2500 and 1350, respectively ( $p$-value $<0.0001)$, the difference did not exceed one logarithm, and therefore is not relevant for Legionella risk assessment. These results make questionable the need to utilize BCYE agar plates to analyze potable water samples.
\end{abstract}

Keywords: Legionella; Legionnaires' disease; culture; BCYE and GVPC media

\section{Introduction}

Legionella is a water-born pathogen widely spread in man-made water systems, responsible for a severe pneumonia and a flu-like illness, named Legionnaires' disease (LD) and the Pontiac fever, respectively. Overall, at the present time, 62 Legionella spp. have been identified and less than a half were pathogenic, however, Legionella pneumophila was surely the most frequently found in LD cases. 
After its first isolation following the large outbreak in Philadelphia in 1976, Legionella has become an opportunistic pathogen of major concern, because of a worldwide increasing number of both sporadic cases and outbreak events [1-4]. Outbreak investigations have widely demonstrated that the most frequent sources of infection are water systems of different buildings, such as hotels or hospitals and, specifically, showers, cooling towers, and spa pools $[5,6]$.

The timely identification of the source of an infection is of great importance to prevent clusters or outbreaks and culture examination is the gold standard for the analyses of water samples. Although molecular methods have been demonstrated to be highly sensitive and specific, as well as able to detect all Legionella species and serogroups, they remain impracticable for Legionella enumeration because they detect DNA of both living and dead bacteria [7,8].

The Legionella laboratory isolation is of great relevance for further deeper molecular investigations, in order to characterize clinical and environmental strains and identify the source of infection [9]. Furthermore, according to the European guidelines for Legionella [10], a quantitative evaluation of the contamination of water systems due to Legionella can be determined only by a culture, even though a culture has been demonstrated to have some drawbacks [11]. The counting of colony forming units per liter (CFU/L) is a crucial step for risk assessment and, as a consequence, to decide the right control measures to be adopted. The fastidious growth requirements of Legionella, the overgrowth of other bacteria, as well as the medium required by the specifically adopted culture method can affect the results of the analysis and determine a variable range of Legionella concentrations.

Culture methods are generally performed according to standards, such as the international standard organization (ISO), recognized by each country's accreditation body [12]. In particular, the ISO 11731 is the most used and it has recently been updated, replacing the previous ones published in 1998 and 2004 (ISO 11731: 2017). Chemical formulation of culture media, as well as the acid and heat treatments, required by the ISO 11731 for the enumeration of Legionella in water samples, may have different effects on the recovery of Legionella, independent of the manufacturer of the commercially available media $[13,14]$.

The updated ISO 11731 introduced the utilization of the following three media: the buffered charcoal yeast extract (BCYE) agar; the BCYE with selective supplements (BCYE $+A B)$, containing polymixin $\mathrm{B}$, sodium cefazolin, and pimaricin; and the highly selective Modified Wadowsky Yee (MWY) agar or, as an alternative, the glycine, vancomycin, polymyxin B, cycloheximide (GVPC) agar. The choice of the selective medium to be used is linked to the specific potential bacterial contamination of water samples. Potable waters and any other water samples with background microorganisms must be analyzed using selective media, with the capability of reducing background microorganisms. In the ISO 11731: 2017, GVPC and MWY are both considered to be equally efficient for Legionella recovery. It has been demonstrated that MWY was the best medium for isolating Legionella pneumophila from potable water samples and GVPC was the most effective for reducing additional microbial flora $[15,16]$. According to ISO 11731: 2017, the decision matrix in Annex J shows that plating on BCYE agar is specifically required for potable water samples when the enumeration of Legionella is determined by the following methods: (i) direct plating without any concentration and treatment, (ii) membrane filtration and direct placing of the membrane filter on culture media, and (iii) membrane filtration followed by washing procedure.

In this study, the Legionella recovery from potable water samples, concentrated by filtration with washing procedure, was determined and the recovery after plating on BCYE and GVPC agar plates was compared.

\section{Material and Methods}

\subsection{Water Samples and Culture}

Overall, 556 water samples were collected from accommodation sites, hospitals, and private homes and, according to the ISO 11731: 2017, they were classified as belonging to the identified Matrix 
A, being water samples expected at low concentration of interfering microorganisms. Sampling was performed at different sampling points (shower, faucet, boiler, etc.) of accommodation sites and hospitals according to protocols reported in Italian guidelines [17]. Briefly, an instant water sample was collected to simulate exposure by a user, without flaming and disinfecting the outlet, and without running water. The temperature was measured immediately before filling the one-liter bottle.

The BCYE and GVPC (Oxoid, Thermo Fisher Diagnostics Limited, Cheshire, UK) agar plates and reagents were prepared according to ISO 11731: 2017. Legionella CYE agar base, BCYE- $\alpha$ growth supplement, and GVPC selective supplement were purchased from Oxoid, Thermo Fisher Diagnostics Limited, Cheshire, UK. For each lot of both media, a quality control was carried out according to ISO 11133: 2014 [18]. The reference material Easy-tab Reference Material (LGC, Bury, UK) was utilized for performance testing of the two media, and always resulted within the declared range. Selectivity of GVPC was qualitative determined according to ISO 11133: 2014. Ringer solution was used to wash polycarbonate membranes using a vortex mixer.

For each sample, a volume of one liter was collected and it was concentrated 200 times by filtration, using $0.22 \mu \mathrm{m}$ polycarbonate membrane, followed by the washing procedure of the filter. After filtration the membrane was placed in a screw cap sterile container with $5 \mathrm{~mL}$ of diluted Ringer's solution. The membrane was washed by shaking vigorously for at least $2 \mathrm{~min}$ using a vortex mixer. The concentrated sample was divided into the following three aliquots, according to ISO 11731: 2017: one $\mathrm{ml}$ was heat treated, one $\mathrm{ml}$ was acid treated, and the remaining $3 \mathrm{~mL}$ were untreated. Then, one hundred $\mu \mathrm{L}$ of each aliquot were placed on both BCYE and GVPC agar plates and incubated at $37^{\circ} \mathrm{C}$, for ten days. The plates were checked after four or five days, and after 10 days. According to the concentration procedure, the detection limit of our method was $50 \mathrm{CFU} / \mathrm{L}$.

The laboratory that analyzed the samples is accredited for the detection and enumeration of Legionella according to ISO 11731: 2017, by the Italian national accreditation body (Accredia).

\subsection{Statistical Analysis}

The McNemar's test was used to compare frequency on paired data. The concordance between media was evaluated using the Kappa test $(\mathrm{K}<0.20=$ "poor", $0.20-0.40=$ "fair", $0.40-0.60=$ "moderate", $0.60-0.80=$ "good", and 0.80-1.00 = "very good"). Specificity and sensitivity, as well positive and negative predictive values (PPV and NPV, respectively), and 95\% confidence intervals (CI) for both media were calculated, considering the BCYE as a reference medium.

All statistical analyses were performed by Stata software version 11.2 (Stata Corp, College Station, TX, USA).

\section{Results}

All of the 556 water samples were analyzed by culture and, overall, $151(27.1 \%)$ were positive for Legionella, of which 65 (43\%) grew on only GVPC, 85 (56.3\%) grew on both GVPC and BCYE, and one $(0.7 \%)$ grew on only BCYE. (Table 1$)$. The difference of the results obtained analyzing the samples by the two media was significant (McNemar's test, $p<0.0001$ ).

Table 1. Recovery of Legionella by using buffered charcoal yeast extract (BCYE) and glycine, vancomycin, polymyxin B, cycloheximide (GVPC) media.

\begin{tabular}{|c|c|c|c|c|}
\hline \multicolumn{5}{|c|}{ GVPC } \\
\hline & & Negative & Positive & Total \\
\hline \multirow{3}{*}{ BCYE } & Negative & 405 & 65 & 470 \\
\hline & Positive & 1 & 85 & 86 \\
\hline & Total & 406 & 150 & 556 \\
\hline
\end{tabular}


The sensitivity, specificity, positive predictive value (PPV), and negative predictive value (NPV) of GVPC vs. BCYE media are shown in Table 2. GVPC demonstrated a greater sensitivity and a good specificity as compared with BCYE medium. The Cohen's Kappa coefficient calculated on these data provided a value of 0.65 , indicating a good quality of agreement $(p<0.0001)$ (Table 2$)$.

Table 2. GVPC vs. BCYE sensitivity, specificity, positive predictive value (PPV), negative predictive value (NPV), concordance, Kappa value, and $p$-value.

\begin{tabular}{cc}
\hline Comparison & $\mathbf{\%}(\mathbf{9 5} \% \mathbf{C I})$ \\
\hline Sensitivity & $98.8(97.9-99.7)$ \\
Specificity & $86.2(83.3-89.0)$ \\
PPV & $56.7(52.5-60.8)$ \\
NPV & $99.7(99.3-100.2)$ \\
Concordance & 88.1 \\
Kappa value $(p$-value $)$ & $0.65(<0.0001)$
\end{tabular}

$\mathrm{CI}$, confidence interval; $\mathrm{PPV}$, positive predictive value; NPV, negative predictive value.

Considering the 85 samples that were positive on both GVPC and BCYE media, 11 samples showed fewer colony forming units per liter (CFU/L) on GVPC than on BCYE (Table 3). However, the differences of CFU/L found between the two media were never higher than $1 \log (p$-value $=0.0388)$.

Table 3. Legionella CFU/L range detected on samples positive on both BCYE and GVPC media.

\begin{tabular}{cccccc}
\hline & & \multicolumn{3}{c}{ GVPC } & Total \\
& CFU/L & $\mathbf{5 0 - 1 0 0 0}$ & $\mathbf{1 0 5 0 - 1 0 , 0 0 0}$ & $\mathbf{> 1 0 , 0 0 0}$ & \\
\hline \multirow{3}{*}{ BCYE } & $50-1000$ & 30 & 3 & 0 & 33 \\
& $1050-10,000$ & 5 & 33 & 0 & 38 \\
& $>10,000$ & 0 & 6 & 8 & 14 \\
& Total & 35 & 42 & 8 & 85 \\
\hline
\end{tabular}

Figure 1 shows the distribution of L. pneumophila serogroups and Legionella species found on GVPC and BCYE agar plates. Legionella species were detected in 19 samples by GVPC and in 13 samples by BCYE. Considering the range of the colony forming unit (CFU)/L detected, the data proved that, among the 65 samples positive on only GVPC, there were 51 samples $(78.4 \%)$ showing a range between 50 and 1000, highlighting the high efficiency of GVPC in isolating low bacterial counts. The calculated medians of $\mathrm{CFU} / \mathrm{L}$ detected on BCYE and GVPC plates were equal to 2500 (interquartile range $=5500$ ) on BCYE and 1350 (interquartile range $=3950$ ) on GVPC, and the difference between the two media was significant $(p$-value $<0.0001)$.

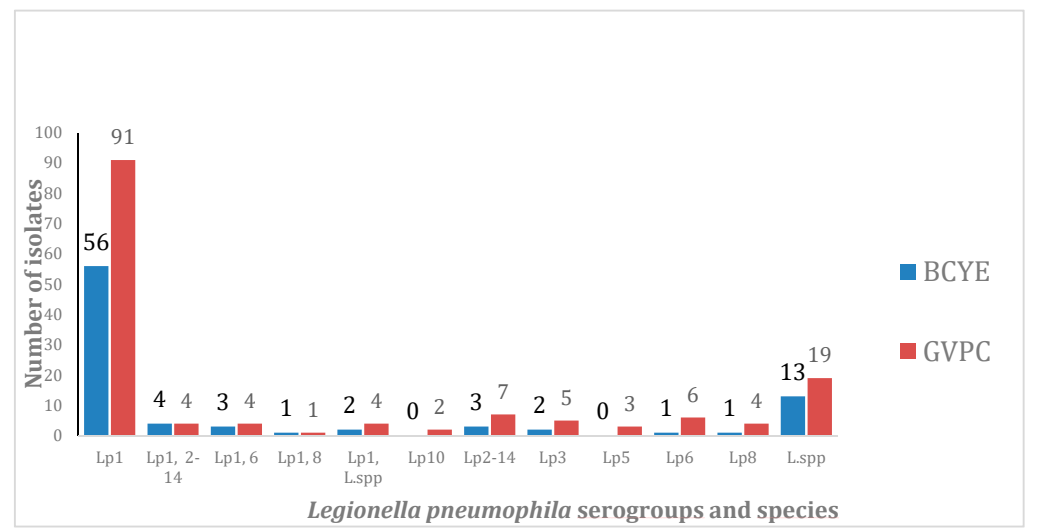

Figure 1. Distribution of Legionella pneumophila serogroups and Legionella species (BCYE $\mathrm{n}=86$ vs. GVPC $n=150$ ). 


\section{Discussion}

In this study, potable water samples were analyzed according to the ISO1173: 2017, in order to compare the Legionella recovery obtained by plating on BCYE and GVPC agar plates. We observed that GVPC was more efficient in detecting Legionella than BCYE medium. Indeed, $43 \%$ of the overall positive samples were detected on only GVPC agar plates and, in addition, the positivity of water samples at low bacterial counts, corresponding to $78.4 \%$ of the total positive samples, was determined only by using this medium. This finding has significant relevance especially when, in specific water systems as hospital wards or thermal waters, the absence or a strong containment of Legionella contamination must be guaranteed, due to possible exposure by people at increased risk of acquiring LD. In a previous study, it was demonstrated that a much greater yield of Legionella spp. was obtained by plating on BCYE than on MWY agar plates, and a significantly higher number of CFU of both Legionella pneumophila and non-pneumophila was counted on BCYE as compared with MWY [19]. On the contrary, Leoni et al. demonstrated the significantly higher yield on GVPC and MWY than on BCYE medium, in combination with the technique used of direct inoculum or pretreatment with acid or heat [18]. Furthermore, other studies have shown no statistically significant differences between BCYE and GVPC media in recovering Legionella in water samples $[14,20]$. In this study, Legionella recovery was determined by comparing the CFU/L counted in BCYE and GVPC, and although significantly higher CFU/L were found in BCYE than in GVPC agar plates, the difference did not exceed one log. Therefore, it was not relevant for Legionella risk assessment of drinking water systems.

Furthermore, the possibility of improving the isolation of Legionella non-pneumophila species by plating the water samples on BCYE was not confirmed. Indeed, Legionella species other than pneumophila were detected more on GVPC than on BCYE.

In conclusion, these results cast doubt on the advantages of analyzing water samples using only BCYE, as required by ISO 11731: 2017. Further investigations by analyzing a larger number of water samples should be conducted to confirm these data, which, if confirmed, would bring enormous benefits, saving time and money, especially for laboratories that carry out monitoring activities and analyze hundreds of samples daily.

Author Contributions: Conceptualization, A.S.; Data curation, E.P., M.M., P.B., A.B., and A.S.; Formal analysis, E.P., M.M., P.B., M.S. (Margit Seeber), E.R., and A.-M.P.; Investigation, E.P., A.G., M.S. (Margit Seeber), E.R., and A.-M.P.; Methodology, M.M., E.P. and P.B.; Software, A.B.; Supervision, A.S.; Validation, A.B. and M.L.R.; Visualization, A.G.; Writing-Original draft, M.S. (Maria Scaturro); Writing-Review and editing, M.C.R. and M.L.R. All authors have read and agreed to the published version of the manuscript.

Funding: This research received no external funding.

Conflicts of Interest: The author declares no conflict of interest.

\section{References}

1. Fraser, D.W.; Tsai, T.R.; Orenstein, W.; Parkin, W.E.; Beecham, H.J.; Sharrar, R.G.; Harris, J.; Mallison, G.F.; Martin, M.S.; McDade, J.E.; et al. Legionnaires' disease: Description of an epidemic of pneumonia. N. Engl. J. Med. 1977, 297, 1189-1197. [CrossRef] [PubMed]

2. Hamilton, K.A.; Prussin, A.J., 2nd; Ahmed, W.; Haas, C.N. Outbreaks of Legionnaires' Disease and Pontiac Fever 2006-2017. Curr. Environ. Health Rep. 2018, 5, 263-271. [CrossRef] [PubMed]

3. Legionnaires' Disease-Annual Epidemiological Report for 2017. Surveillance Report 17 January 2019. Available online: https://www.ecdc.europa.eu/en/publications-data/legionnaires-disease-annualepidemiological-report-2017 (accessed on 16 September 2020).

4. Rota, M.C.; Caporali, M.G.; Bella, A.; Scaturro, M.; Giannitelli, S.; Ricci, M.L. Rapporto annuale sulla legionellosi in italia nel 2018. Not. Ist. Super. Sanità 2019, 32, 7-13.

5. Orkis, L.T.; Harrison, L.H.; Mertz, K.J.; Brooks, M.M.; Bibby, K.J.; Stout, J.E. Environmental sources of community-acquired legionnaires' disease: A review. Int. J. Hyg. Environ. Health 2018, 221, 764-774. [CrossRef] [PubMed] 
6. De Filippis, P.; Mozzetti, C.; Amicosante, M.; D’Alò, G.L.; Messina, A.; Varrenti, D.; Giammattei, R.; Di Giorgio, F.; Corradi, S.; D'Auria, A.; et al. Occurrence of Legionella in showers at recreational facilities. J. Water Health 2017, 15, 402-409. [CrossRef] [PubMed]

7. Collins, S.; Stevenson, D.; Walker, J.; Bennett, A. Evaluation of Legionella real-time PCR against traditional culture for routine and public health testing of water samples. J. Appl. Microbiol. 2017, 122, 1692-1703. [CrossRef] [PubMed]

8. Lee, J.V.; Lai, S.; Exner, M.; Lenz, J.; Gaia, V.; Casati, S.; Hartemann, P.; Lück, C.; Pangon, B.; Ricci, M.L.; et al. An international trial of quantitative PCR for monitoring Legionella in artificial water systems. J. Appl. Microbiol. 2011, 110, 1032-1044. [CrossRef] [PubMed]

9. Phin, N.; Parry-Ford, F.; Harrison, T.; Stagg, H.R.; Zhang, N.; Kumar, K.; Lortholary, O.; Zumla, A.; Abubakar, I. Epidemiology and clinical management of Legionnaires' disease. Lancet. Infect. Dis. 2014, 14, 1011-1021. [CrossRef]

10. European Technical Guidelines for the Prevention, Control and Investigation, of Infections Caused by Legionella Species" June 2017. Available online: https://www.ecdc.europa.eu/en/publications-data/europeantechnical-guidelines-prevention-control-and-investigation-infections (accessed on 16 September 2020).

11. Collins, S.; Jorgensen, F.; Willis, C.; Walker, J. Real-time PCR to supplement gold-standard culture-based detection of Legionella in environmental samples. J. Appl. Microbiol. 2015, 119, 1158-1169. [CrossRef] [PubMed]

12. ISO-ISO 11731: 2017-Water quality—Enumeration of Legionella; ISO: Geneva, Switzerland, 2017.

13. Lück, P.C.; Igel, L.; Helbig, J.H.; Kuhlisch, E.; Jatzwauk, L. Comparison of commercially available media for the recovery of Legionella species. Int. J. Hyg. Environ. Health 2004, 207, 589-593. [CrossRef] [PubMed]

14. Lee, T.C.; Vickers, R.M.; Yu, V.L.; Wagener, M.M. Growth of 28 Legionella species on selective culture media: A comparative study. Clin. Microbiol. 1993, 31, 2764-2768. [CrossRef] [PubMed]

15. Reinthaler, F.F.; Sattler, J.; Schaffler-Dullnig, K.; Weinmayr, B.; Marth, E. Comparative study of procedures for isolation and cultivation of Legionella pneumophila from tap water in hospitals. J. Clin. Microbiol. 1993, 31, 1213-1216. [CrossRef] [PubMed]

16. Edelstein, P.H. Comparative study of selective media for isolation of Legionella pneumophila from potable water. J. Clin. Microbiol. 1982, 16, 697-699. [CrossRef] [PubMed]

17. Linee Guida per La Prevenzione Ed Il Controllo Della Legionellosi. 2015. Available online: http://www. salute.gov.it/imgs/C_17_pubblicazioni_2362_allegato.pdf (accessed on 14 September 2020).

18. ISO-ISO 11133: 2014-Microbiology of Food, Animal Feed and Water-Preparation, Production, Storage and Performance Testing of Culture Media; Amendments 1:2018 and 2:2020 included; ISO: Geneva, Switzerland, 2014.

19. Ditommaso, S.; Gentile, M.; Giacomuzzi, M.; Zotti, C.M. Recovery of Legionella species from water samples using an internal method based on ISO 11731: Suggestions for revision and implementation. Diagn. Microbiol. Infect. Dis. 2011, 70, 200-206. [CrossRef]

20. Leoni, E.; Legnani, P.P. Comparison of selective procedures for isolation and enumeration of Legionella species from hot water systems. J. Appl. Microbiol. 2001, 90, 27-33. [CrossRef] [PubMed]

(C) 2020 by the authors. Licensee MDPI, Basel, Switzerland. This article is an open access article distributed under the terms and conditions of the Creative Commons Attribution (CC BY) license (http://creativecommons.org/licenses/by/4.0/). 